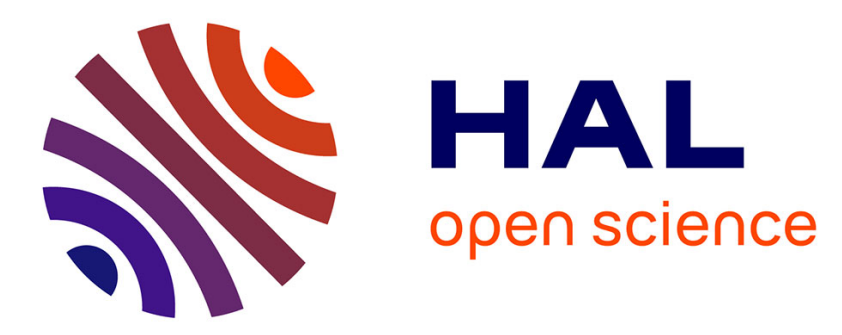

\title{
Unilateral vestibular deafferentation impairs embodied spatial cognition
}

Diane Deroualle, Liliane Borel, Brandon Tanguy, Laurence Bernard-Demanze, Arnaud Devèze, Marion Montava, Jean-Pierre Lavieille, Christophe Lopez

\section{- To cite this version:}

Diane Deroualle, Liliane Borel, Brandon Tanguy, Laurence Bernard-Demanze, Arnaud Devèze, et al.. Unilateral vestibular deafferentation impairs embodied spatial cognition. European Journal of Neurology, 2019, 1, 10.1007/s00415-019-09433-7 . hal-02553545

\section{HAL Id: hal-02553545 \\ https://hal-amu.archives-ouvertes.fr/hal-02553545}

Submitted on 24 Apr 2020

HAL is a multi-disciplinary open access archive for the deposit and dissemination of scientific research documents, whether they are published or not. The documents may come from teaching and research institutions in France or abroad, or from public or private research centers.
L'archive ouverte pluridisciplinaire HAL, est destinée au dépôt et à la diffusion de documents scientifiques de niveau recherche, publiés ou non, émanant des établissements d'enseignement et de recherche français ou étrangers, des laboratoires publics ou privés. 


\section{Unilateral vestibular deafferentation impairs}

\section{2 embodied spatial cognition}

3

4

5 Diane Deroualle ${ }^{1}$, Liliane Bore ${ }^{1}$, Brandon Tanguy ${ }^{1}$, Laurence Bernard-Demanze ${ }^{2}$,

6 Arnaud Devèze ${ }^{3}$, Marion Montava ${ }^{2}$, Jean-Pierre Lavieille ${ }^{2}$, and Christophe Lopez ${ }^{1(*)}$ 7

8

$9 \quad{ }^{1}$ Aix Marseille Univ, CNRS, LNSC, FR3C, Marseille, France

$10{ }^{2}$ Department of Otorhinolaryngology, Head and Neck Surgery, Hôpital La Conception, 11 APHM, Marseille, France

$12{ }^{3}$ Ramsay Générale de Santé, Hôpital Privé Clairval, Department of Otorhinolaryngology, 13 Head and Neck Surgery, Marseille, France

\section{AUTHORS' ORCID:}

Diane Deroualle $=\underline{\text { orcid.org } / 0000-0001-5710-9419}$

Marion Montava = orcid.org/0000-0003-1347-9412

Christophe Lopez $=\underline{\text { orcid.org/0000-0001-9298-2969 }}$

$\left.{ }^{*}\right)$ Address for correspondence:

Dr Christophe Lopez

Laboratoire de Neurosciences Intégratives et Adaptatives - UMR 7260

Aix Marseille Univ \& Centre National de la Recherche Scientifique (CNRS)

Centre Saint-Charles, Fédération de Recherche 3C - Case B

3, Place Victor Hugo

13331 Marseille Cedex 03, France

Tel: +33413550841 Fax: + 33413550844

email: christophe.lopez@univ-amu.fr 


\section{Abstract}

36 A growing number of studies indicate that cognitive complaints are common in patients with peripheral 37 vestibular disorders. A better understanding of how vestibular disorders influence cognition in these 38 patients requires a clear delineation of the cognitive domains affected by vestibular disorders. Here, we 39 compared the consequences of left and right vestibular neurectomy on third-person perspective taking 40 - a visuo-spatial task requiring mainly own-body mental imagery, and on 3D objects mental rotation 41 imagery - requiring object-based mental imagery but no perspective taking. Patients tested one week 42 after a unilateral vestibular neurectomy and a group of age- and gender-matched healthy participants 43 played a virtual ball-tossing game from their own first-person perspective (1PP) and from the 44 perspective of a distant avatar (third-person perspective, 3PP). Results showed larger response times in 45 the patients with respect to their controls for the 3PP taking task, but not for the 1PP task and the 3D 46 objects mental imagery. In addition, we found that only patients with left vestibular neurectomy 47 presented altered 3PP taking abilities when compared to their controls. This study suggests that 48 unilateral vestibular loss affects mainly own-body mental transformation and that only left vestibular 49 loss seems to impair this cognitive process. Our study also brings further evidence that vestibular signals 50 contribute to the sensorimotor bases of social cognition and strengthens the connections between the so 51 far distinct fields of social neuroscience and human vestibular physiology.

\section{Keywords}

56 Perspective taking, Vestibular system, Unilateral vestibular loss, Mental imagery, 3D object mental 57 rotation 


\section{Introduction}

A growing number of studies indicate that cognitive complaints are common in patients with vestibular disorders (reviewed in $[1,2]$ ). For example, a recent epidemiological study in US adults revealed that patients with vestibular vertigo have an eight-fold increase in the odds of reporting impaired memory and attention, limiting their activities [3]. A better understanding of how vestibular disorders influence cognition in these patients requires a clear delineation of the cognitive domains affected by vestibular disorders. Yet, the paucity of publications reporting negative findings makes it difficult to identify the cognitive domains impaired and spared in patients with vestibular disorders.

Several studies have compared populations of patients with vestibular disorders and controls, with mixed findings depending on the aetiology, on unilateral vs bilateral vestibular loss, history of the disease and the cognitive task. Popp et al. [4], for example, showed that bilateral vestibular loss impaired visuospatial abilities, processing speed, short-term memory and executive functions, whereas unilateral vestibular loss impaired only visuospatial abilities and processing speed. Spatial memory was impaired in navigation studies using locomotion [5] and virtual reality tasks [6]. Vestibular disorders also impaired space representation when participants were not moving or displaced at all, such as during mental rotation of bodies and non-corporeal objects [7-10]. We note that it remains unclear from these studies if vestibular disorders impair more egocentric (i.e. own-body) mental imagery or object-based mental imagery.

Another debated question concerns the consequences of left $v s$ right peripheral vestibular disorders on cognition. A rationale for this question is the anatomical and functional asymmetry within the vestibulo-thalamo-cortical system. Neuroimaging studies have reported a predominance of vestibular processing in the right cerebral hemisphere in right-handed participants, and an overall dominance of the projections from one labyrinth to the ipsilateral cerebral hemisphere $[11,12]$. As various cognitive tasks involve differently lateralized brain areas, different effects of left and right vestibular disorders on cognition can be predicted. Only few studies have tackled directly this question, and overall their findings were controversial, showing stronger impairments in left [13] or right [14] vestibular disorders, or equal impairments in left and right vestibular disorders [4].

The present study aims at clarifying the vestibular contributions to visuo-spatial perspective taking. While the environment is usually experienced from an egocentric first-person perspective (1PP), humans can simulate someone else's viewpoint, referred to as third-person perspective (3PP) taking. 3PP taking is an important component of social cognition which contributes to understand others and anticipate their actions. There is evidence suggesting that 3PP taking is a strongly embodied process involving egocentric mental imagery [15]. Here, we compared patients one week after right or left vestibular neurectomy and healthy participants involved in a virtual ball-tossing game $[16,17]$ played either from their own viewpoint (1PP), or from the viewpoint of a distant avatar - thus requiring 3PP taking. Patients were tested in the acute stage of a unilateral vestibular deafferentation, when brain 
metabolism disorganization is maximal [18], to document the impact of the vestibular tone imbalance on 3PP taking abilities. Performances were compared to those from 3D objects mental rotation, requiring object-based mental imagery, but no perspective-taking. Given evidence of a vestibular contribution to the perspectival experience $[16,19,20]$, and overlapping neural mechanisms for vestibular information processing and perspective-taking [21], we hypothesized that unilateral vestibular deficits would impair 3PP taking more than object-based mental imagery.

\section{Methods}

\section{Patients}

We tested 23 patients ( 13 women; mean age \pm SD: $51 \pm 12$ years) before and 7 days after a unilateral vestibular neurectomy. Cognitive tests were performed 7 days after the surgery. All patients were righthanded (Edinburgh Handedness inventory: $79 \pm 22 \%$ ). Twenty-one patients were operated on for an acoustic neuroma (grade 2, 3 or 4 from Koos classification [22]) and two patients for Menière's disease (Table 1). Menière's disease patients had a pure unilateral vestibular pathology, with normal video head impulse test (vHIT) and hearing on their contralesional ear. Twelve patients received a left neurectomy and 11 a right neurectomy using the retrosigmoid approach $(n=20)$ or the translabyrinthine approach $(n=3)$. Depending on the size of the neuroma and the operating approach, the neurectomy aimed at removing the vestibular nerve totally $(n=19)$ or partially $(n=4)$. Before and 7 days after neurectomy, patients received standard otoneurological examinations including a pure tone audiometry (SCR Electroniques, France), a bithermal caloric test and a vHIT (Synapsys, France), as well as measurement of vestibulo-ocular responses on a rotating chair (Synapsys, France) and of the subjective visual vertical (Framiral, France). Patients were compared to 23 age- and gender-matched healthy volunteers (13 women, $52 \pm 12$ years). They were right-handed $(86 \pm 25 \%)$ and had no history of otoneurological and psychiatric disease.

\section{Ethics statement}

All participants were informed about the study and gave their written informed consent. Experimental procedures were approved by the local Ethics Committee (CPP Sud-Méditerranée II, 2011-A01221-40) and followed the Declaration of Helsinki.

\section{Visual stimuli and tasks}

3PP taking task. Participants were involved in a virtual ball-tossing game [16]. Visual stimuli showed 6 avatars around a circle (see Figure 1A and the legend, for details). Participants were instructed to adopt the perspective of the avatar holding the ball (identified by a blue T-shirt) and from this position to toss the ball to the avatar wearing a red T-shirt. They indicated as quickly and as accurately as possible 
130 whether they had to toss the ball to the right or left, by pressing on a respond pad [16]. There was no time limit to answer. The experiment was a 5 Angle of visuo-spatial perspective taking (5 positions of the avatar holding the ball: $\pm 60^{\circ}, \pm 120^{\circ}$ and $\left.180^{\circ}\right) \times 4$ positions of the avatar receiving the ball $\left( \pm 60^{\circ}\right.$ and $\pm 120^{\circ}$ with respect to the avatar holding the ball) design, with four repetitions of each of the 20 stimuli. Participants completed 80 trials divided into 4 blocks of 20 trials presented in a random order.

1PP task. Five avatars were shown in the visual scene (Figure 1B) and the hands holding the ball in the front of the scene indicated that participants had to toss the virtual ball from their 1PP [16]. Participants indicated as quickly and accurately as possible whether they had to toss the ball to their right or left (to the avatar located at $\pm 60^{\circ}, \pm 120^{\circ}$ ) without time limit. Each of the four visual stimuli was repeated four times in one block of 16 trials presented in a random order.

Visual detection task. A visual detection task [16] was used to control for potential attentional and visual differences between groups of participants (Figure 1C). In 10 trials, one avatar held a ball, while in 10 other trials, no ball was shown. Participants were instructed to detect as quickly and as accurately as possible that there was a ball in the visual scene, without time limit. Participants completed one block of 20 trials presented in a random order.

3 D objects mental rotation task. Twenty out of 23 patients were involved in a 3D objects mental rotation task [9]. Pairs of identical or different 3D objects were presented side by side on a computer screen (Figure 1D). When identical, the second object was rotated with respect to the first object by $20^{\circ}, 40^{\circ}$ or $80^{\circ}$. We instructed participants to imagine a rotation of one of the objects to align it with the other and to indicate as quickly and as accurately as possible whether the objects were identical or different. There was no time limit to answer. There were two blocks of 36 pairs of objects, of which 24 were identical and 12 were different. Pairs of objects were presented randomly within each block, and the presentation of the blocks was counterbalanced.

\section{Experimental procedures}

Participants were tested while comfortably sitting on a chair with their head maintained by a forehead and chin rest. SuperLab 4.5 (Cedrus Corporation, USA) was used to present visual stimuli on a computer screen $(64.4 \times 49.4 \mathrm{~cm})$ placed $50 \mathrm{~cm}$ in front of the participants. Thirteen patients and controls first completed the 1PP task and then the 3PP taking task, while the others started with the 3PP taking task. All participants finished with the visual detection task and the $3 \mathrm{D}$ objects mental rotation task. Participants took short breaks after each block and task. Before the experiment, they trained to each task with a random selection of 10 trials. After the experiment, participants filled out a questionnaire about the strategies they used. They indicated whether it happened during the experiment they imagined 
rotating the entire visual scene with the avatars to solve the 3PP taking task. If it was the case, they were asked to rate how often they did it, using a 7-point Likert scale ranging from 'rarely' to 'all the time'.

\section{Data analysis}

For each participant and task, we calculated the mean response times (RTs) and percentage of errors. Trials yielding incorrect answers were discarded from the analysis of the RTs and we excluded trials for which RTs exceeded 2 standard deviations of the participant's grand average. As the Shapiro-Wilk test revealed not normally distributed RTs for most of the dependent variables, we used a log transformation and applied repeated-measures ANCOVA when this procedure led to normal distribution. Variables that remained non-normally distributed and the percentage of errors were analysed using non-parametric procedures (SPSS 25, IBM Corporation, USA). Differences were considered significant when $P<0.05$. One outlier participant was excluded for the 3PP taking task (RTs $>16 \mathrm{~s}$ ), as well as one participant for the $1 \mathrm{PP}$ and visual detection tasks, resulting in a total of 22 patients and 22 controls in the final analysis.

We used partial correlations to calculate the relation between 13 clinical parameters (Table 1) and performance in the cognitive tasks. To minimize the risk of false positives, we used Bonferronicorrected $\alpha$ level of $P<0.05 / 13$ for statistical significance $(P<0.0039)$.

\section{Results}

\section{PP taking task}

Response times. RTs were first analysed using a repeated-measures ANCOVA with Angle of 3PP taking as within-subject factor, Group and Gender as between-subject factors, and Age as covariate. Age was significantly related to RTs $\left(\mathrm{F}_{1,39}=12.19, P<0.005\right)$, whereas Gender was not $\left(\mathrm{F}_{1,39}=0.08, P=0.77\right)$. Importantly, we found a significant effect of the Group after controlling for the effect of Age $\left(\mathrm{F}_{1,39}=\right.$ 4.40, $P<0.05$ ). Figure $2 \mathrm{~A}$ shows significantly larger RTs for 3PP taking in patients than in controls. As expected, the analysis shows a significant effect of the Angle of 3PP taking $\left(\mathrm{F}_{3,107}=2.78, P<0.05\right)$, with longer RTs for larger angles of perspective taking (Greenhouse-Geisser correction, $\varepsilon=0.69$ ). There was no significant interaction between Angle and Group, or with the covariate. RTs were not significantly related to the grade of the neuroma (Kruskal-Wallis test, $P>0.05$ ) (Figure 2B).

In a second analysis, we explored how the side of neurectomy influenced 3PP taking. We found that patients with left neurectomy showed significantly larger RTs than their age and gender-matched controls $\left(\mathrm{F}_{1,17}=6.81, P<0.05\right)$ (Figure 2A). There was no other significant main effect or interaction, but Age was significantly related to RTs $\left(F_{1,17}=8.04, P<0.05\right)$. By contrast, patients with right neurectomy did not differ from their controls $\left(\mathrm{F}_{1,17}=0.04, P=0.84\right)$ (Figure 2A). The effect of Angle of 3PP taking was not significant after Greenhouse-Geisser correction $\left(\mathrm{F}_{2,38}=2.53, P=0.09\right)$, but the 
effect of Age was significant $\left(\mathrm{F}_{1,17}=7.74, P<0.05\right)$. Direct comparison of patients' performance with left and right neurectomy did not reveal significant differences in RTs $\left(\mathrm{F}_{1,17}=2.42, P=0.14\right)$. Thus, the effect of Group observed in the whole-population analysis seems to arise from altered 3PP taking mainly in patients with left neurectomy. We note that this result cannot be accounted for by different vestibular functions in patients with left and right neurectomy (see Table 1).

Errors. Friedman's tests revealed an effect of the Angle of 3PP taking on the percentage of errors in patients $\left(\chi_{(4)}^{2}=25.7, P<0.001\right)$ and controls $\left(\chi_{(4)}^{2}=37.8, P<0.001\right)$ (Figure 2C). However, MannWhitney tests did not reveal a significant effect of the Group $\left(-60^{\circ}: P=0.13 ;-120^{\circ}: P=0.16 ; 180^{\circ}: P\right.$ $\left.=0.33 ;+120^{\circ}: P=0.44 ;+60^{\circ}: P=0.08\right)$.

Questionnaire about 3PP taking strategies. 18/22 patients (82\%) and 19/22 controls (86\%) reported they never imagined rotating the entire visual scene to solve the task, whereas $4 / 22$ patients $(18 \%)$ and $3 / 22$ controls (14\%) reported they "sometimes" imagined rotating the visual scene. No participant reported having used this strategy "all the time". Strategies were reported in similar proportion in the whole sample of patients and controls $\left(\chi^{2}=0.17, P=0.68\right)$, and in similar proportion in patients with left and right vestibular neurectomy $\left(\chi^{2}=1.22, P=0.27\right)$.

\section{First-person perspective task}

RTs between the whole sample of patients and the controls did not differ (Mann-Whitney test, $U=244$, $P=0.55$ ) (Figure 2D). Similar analyses for patients with left and right neurectomy revealed no differences between the groups (left: $U=22, P=0.15$; right: $U=40, P=0.48$ ). As the percentages of errors were very low, they were not analysed.

\section{Visual detection task}

RTs were significantly longer in the whole sample of patients when compared to the controls (MannWhitney test, $U=287, P<0.05$ ) (Figure 2D). RTs differed between the patients with left neurectomy and their controls $(U=113, P<0.05)$, but they were not different between the patients with right neurectomy and their controls $(U=40, P=0.63)$. As the percentages of errors were very low, they were not analysed.

\section{D objects mental rotation}

Response times. A repeated-measures ANCOVA revealed a significant main effect of Angular disparity (Greenhouse-Geisser correction, $\varepsilon=0.67: \mathrm{F}_{1.3,47}=18.95, P<0.0001$ ), with longer RTs for larger angles of mental rotation. In contrast with the 3PP taking task, there was no significant effect of the Group $\left(\mathrm{F}_{1,35}=0.15, P=0.71\right)$ (Figure 3A). We found no significant effect of Age $\left(\mathrm{F}_{1,35}=3.07, P=0.09\right)$ and 
238 Gender $\left(\mathrm{F}_{1,35}=0.47, P=0.50\right)$, but there was a significant interaction of Angular disparity $\times$ Age $\left(\mathrm{F}_{1.3,47}\right.$

$\left.{ }_{2}^{1} 239=3.01, P<0.05\right)$. Similar analyses conducted separately for patients with left and right neurectomy 3240 revealed no effect of the Group (left: $\mathrm{F}_{1,15}=0.09, P=0.78$; right: $\mathrm{F}_{1,15}=0.03, P=0.87$ ) (Figure 3A).

4

Errors. Friedman's tests revealed a significant effect of Angular disparity in patients $\left(\chi_{(2)}^{2}=27.3, P<\right.$ $0.0001)$ and in controls $\left(\chi_{(2)}^{2}=22.7, P<0.0001\right)$, with more errors for larger angles of mental rotation (Figure 3B). Mann-Whitney tests showed a statistical trend for higher percentage of errors in patients than in controls for $20^{\circ}(U=269, P=0.06)$, but not for $40^{\circ}(U=249, P=0.19)$ and $80^{\circ}(U=260, P=$ $0.11)$.

\section{Clinical data and their correlation with visuo-spatial cognition}

Table 1 shows that overall the two groups of patients did not differ with respect to their clinical status, with the exception of worse postural performance in patients with right than left vestibular loss, and a larger proportion of cophosis at D+7 in the patients with left vestibular neurectomy. There was an important variability in the presurgical vestibular functions, owing to the different locations and grades of the neuroma [22]. Thus, we calculated partial correlations between clinical parameters and cognitive performance, while controlling for the effects of age (as RTs for 3PP taking were related to age). There was no significant correlation between clinical parameters and cognitive performance at a Bonferroni-corrected $\alpha$ level. The only statistical trend was a positive correlation between the deficit at the caloric test measured at D-1 and the average RTs (log transform) for the 3PP taking task $(r=0.596, P=0.007)$. No such trend was found for the correlation between the other cognitive tasks and clinical parameters recorded at $\mathrm{D}-1$ and $\mathrm{D}+7$.

\section{Discussion}

\section{Different effect of vestibular disorders on egocentric vs object-based mental imagery}

The present data show that patients tested one week after unilateral vestibular neurectomy had impair 3PP taking abilities, as revealed by longer RTs in patients than in controls. Interestingly, no deficit was found when patients were required to toss the virtual ball from their 1PP, that is, when no own-body mental transformation was necessary. This indicates that deficits in 3PP taking were not merely related to attentional or oculomotor deficits to explore the visual scene and to discriminate left/ right orientations (see below). Rather, our results speak in favour of a more specific vestibular contribution to perspective taking and highlight the specific role of vestibular information in embodied spatial cognition [23]. Another recent study, in which patients with objective balance deficits were required to imitate lateral body tilts of an avatar, showed longer movement latencies and more errors in the patients [7]. Our results 
274 are also in line with a previous study in healthy participants using the same 3PP taking task, showing that natural vestibular stimulation (on a rotating chair) helped to adopt the perspective of a distant avatar when the directions of the physical rotation and simulated viewpoint were congruent [16].

Although our participants were trained to use explicitly own-body mental imagery during the 3PP taking task, we cannot exclude that some of them used other strategies, such as simulating a 'bird perspective' of the visual scene or mixed egocentric/allocentric strategies depending on the avatar's position. Debriefing questionnaires confirmed that most participants complied to the instructions and used mainly an egocentric strategy. Importantly, as mixed egocentric/allocentric strategies were reported in similar proportions in the patients and the controls, strategies cannot account for the different $3 \mathrm{PP}$ taking abilities between the groups.

In contrast with the 3PP taking task, the unilateral vestibular neurectomy had no effect on 3D object mental imagery. There are only few studies about the consequences of vestibular disorders on object-based mental imagery and they showed divergent results. Impaired object-based mental imagery seems to depend on the extent of the vestibular loss, as patients with bilateral loss were the most impaired $[8,9]$, and on the compensation stage, as deficits were found only in the early stage after vestibular neurectomy $[9,10]$.

Altogether, our data suggest that acute unilateral vestibular deficits affect differently visuospatial tasks involving mostly egocentric mental imagery (3PP taking) and object-based mental imagery. We propose that shared neural networks are involved in encoding own-body rotations (vestibular cortical network) and in simulating own-body and viewpoint rotations (3PP taking). This proposition is first supported by our previous results in healthy participants showing that whole-body rotations on a rotating chair influenced 3PP taking in a direction-specific way, but not object-based mental imagery [16]. Second, a recent fMRI study [21] showed that clockwise/counterclockwise mental rotation of the observer's viewpoint (as used in our 3PP taking task) and galvanic vestibular stimulation both activated the bilateral posterior insular cortex and the right parietal operculum (OP2), two core areas of the vestibular cortical network.

\section{Different effect of right $v s$ left vestibular disorders on perspective taking}

A main result of the present study is that 3PP taking abilities depended on the side of vestibular deafferentation. Only patients with left neurectomy showed larger RTs in the 3PP taking task, when compared to their controls. This was not the case when visuo-spatial judgements were performed from their 1PP. However, patients with left neurectomy were also slower for the visual detection task. This indicates that left vestibular loss is more likely to disturb the mechanisms of egocentric own-body mental imagery and alertness than the mechanisms of visuo-spatial tasks that do not require egocentric mental imagery (i.e., 1PP task and 3D object mental rotation). It is difficult to compare our data with previous studies as only few of them have investigated spatial cognition with respect to the side of the vestibular loss. In line with our findings, Saj et al. [13] found that only left vestibular neurectomy significantly 
311 deviated the perceived straight ahead. Left cathodal galvanic vestibular stimulation also impaired mental

1312

3313

4 imagery in healthy participants [24], while only left-warm caloric vestibular stimulation distorted their body schema [25]. However, opposite data have been reported, with slower recovery of the subjective visual vertical in right vestibular neuritis [26], and a trend for poorer spatial navigation in right vestibular deficits [14]. Another study reported no effect of the side of the vestibular loss on cognitive deficits [4]. The discrepancy between these data may be related to the diversity of the spatial functions tested, involving different brain areas.

Why would left vestibular neurectomy impair 3PP taking? Neuroimaging studies have emphasized a right hemispheric dominance of vestibular processing in right-handed participants [11] as were our participants. Accordingly, a stronger impact of right vestibular neurectomy on 3PP taking can be expected. Our results do not support this hypothesis. However, they are in line with neuroimaging data showing different compensation patterns in acute right and left vestibular neuritis, with a shift of the dominant ascending input from the ipsilateral to the contralateral pathways [18]. Alternatively, based on results from a diffusion tensor imaging study of the vestibulo-thalamo-cortical pathways [12], we predict that right vestibular neurectomy would predominantly disrupt multisensory processing in the right parieto-insular cortex, whereas left neurectomy is more likely to disrupt multisensory processing bilaterally in this area. We propose that left vestibular neurectomy evoked deficits in 3PP taking by disrupting bilateral areas underpinning 3PP taking. Of note, an fMRI study [17] described the neural correlates of 3PP taking in a virtual ball-tossing game similar to that used in the present study. 3PP taking, when compared to 1PP, activated bilaterally the anterior insula and medial frontal cortices, as well as the left inferior parietal lobe. As 3PP taking involves a bilateral network of insular and parietofrontal areas, we anticipate that it is very likely more affected by predominantly bilateral vestibulothalamo-cortical projections from the left vestibular receptors [12].

\section{Role of visuo-spatial attention and alertness}

Patients had longer RTs than controls in the visual detection task, and RTs for the 3PP taking task and the visual detection task were larger after left than right vestibular neurectomy. As previous studies have related vestibular loss to poorer visuo-spatial ability and attention [27], we cannot exclude that larger attentional deficits in patients with left neurectomy contributed to larger RTs for 3PP taking. However, as RTs were not larger for the 1PP task and the 3D-object mental imagery task in these patients, we can rule out a general effect of attentional deficits in all tasks after left neurectomy. We interpret increased RTs in the visual detection task as resulting from deficits of alertness (because RTs were short, around $750 \mathrm{~ms}$, whereas RTs were around $3000 \mathrm{~ms}$ for 3PP taking). Visual detection assessed more alertness (reaction times) than high-level visuo-spatial cognition. Our results are consistent with data showing that patients with unilateral vestibular loss performed worse than healthy controls in a processing speed task [4]. 
We only found a statistical trend for slower 3PP taking in patients with larger deficits at the presurgical caloric test. This relation suggests that information from the lateral semicircular canals, or the normal functioning of the structures processing this information, is involved in own-body mental rotation in the yaw plane. The caloric test delivers low-frequency stimulation of the canals and assesses vestibular pathways that are likely relevant for the cognitive functions tested here. Interestingly, stimulation of the lateral canals by whole-body rotation influenced RTs for 3PP taking in a direction-dependent manner [16] and caloric vestibular stimulation improved own-body mental imagery in healthy participants [28]. Our data are congruent with observations in patients with unilateral vestibular disorders showing that lower caloric response correlated with deficits in processing speed, memory and executive function [4]. Finally, we found no correlation between hearing loss and performance in the cognitive tasks, congruent with recent findings showing similar performance in deaf signers and hearing nonsigners engaged in visuo-spatial perspective-taking, 3D object mental rotation and spatial orientation tasks [29].

\section{Limitations of the study and perspectives}

A limitation of the present study is the absence of detailed neuropsychological evaluation of the participants, which could have accounted for some variability in our data. However, the mean age in our sample was not high, controls were selected to carefully match the patients, and our statistical analyses controlled for the effect of age.

The present study documents deficits of spatial cognition only in the acute stage of a unilateral vestibular neurectomy. This was motivated by the need to better understand the domains of cognition affected and spared when a vestibular tone imbalance is present, i.e. when compensatory mechanisms are not fully expressed. There is evidence of brain disorganization in the acute stage of a unilateral vestibular loss, both in humans [18] and animal [30] models. It is unknown if the deficits in 3PP taking reported here vanish when patients have compensated from their unilateral vestibular disorder. Future studies should endeavor to explore the postlesional recovery of spatial cognition deficits after unilateral vestibular deafferentation and compare its time course to that of functional recovery.

As in most studies of this kind, a limitation of the present study is the sample size, which may explain some discrepancies in the literature regarding the effects of left and right vestibular loss on cognition $[4,13,14,26]$, in addition to the variety of tasks and procedures used. Studies remain to be conducted in large samples of patients to determine the effects of left $v s$ right vestibular loss on a large range of spatial cognitive functions.

\section{Conclusions}

The present study shows that acute unilateral vestibular deafferentation impairs embodied spatial cognition by disrupting visuo-spatial tasks involving mostly egocentric mental imagery (3PP taking), but not object-based mental imagery. These effects were observed only in patients with left vestibular 
loss. We believe that the present investigation helps understand the cognitive complaints of patients with vestibular disorders. Finally, our data brings further evidence that vestibular signals contribute to several sensorimotor bases of social cognition, including 3PP taking, and strengthens the connections between the so far distinct fields of social neuroscience and human vestibular physiology (for a perspective, see [23]).

\section{Acknowledgements}

The research leading to these results has received funding from the People Programme (Marie Curie Actions) of the European Union's Seventh Framework Programme (FP7/2007-2013) under REA grant agreement number 333607 ('BODILYSELF, vestibular and multisensory investigations of bodilyselfconsciousness').

\section{Compliance with ethical standards}

Conflicts of interest. C. Lopez serves as an associate editor of Frontiers in Integrative Neuroscience and guest editor of Frontiers in Neurology.

Ethical standard. Ethical approvals have been obtained from the local ethics committees (Comite de Protection des Personnes Sud-Mediterranée II, 2011-A01221-40) and participants provided informed consent in accordance with the Declaration of Helsinki. 


\section{Figure legends}

Figure 1. Visual stimuli and tasks. (A) Example of a visual scene for the 3PP taking task showing six avatars located around a circle (adapted from Ref. [16]). One avatar was facing the participants $\left(180^{\circ}\right)$, one was seen from the back $\left(0^{\circ}\right)$, two were on their right $\left(60^{\circ}\right.$ and $\left.120^{\circ}\right)$, and two on their left $\left(-60^{\circ}\right.$ and $\left.-120^{\circ}\right)$. From one trial to another, the colour of the avatars' T-shirt could change (white, red or blue). Participants were instructed to simulate the visuo-spatial perspective of the avatar holding the ball (identified by a blue T-shirt) and from this position to toss the ball to the avatar wearing a red T-shirt. The avatar with the blue T-shirt was located either at $\pm 60^{\circ}, \pm 120^{\circ}$ or $180^{\circ}$ (but never at $0^{\circ}$, to avoid confusion with the 1PP task), resulting in a total of 5 angles of 3PP taking. The avatar wearing the red T-shirt was located $60^{\circ}$ or $120^{\circ}$ to the right or left of this avatar. Participants pressed a key on a response pad (RB-830, Cedrus Corporation, San Pedro, USA) with their right middle finger to toss the ball to the right, or their right index finger to toss the ball to the left. A fixation cross was presented during $1 \mathrm{~s}$ before each visual scene. In the example presented, the correct response is to toss the ball to the left. (B) Example of a visual scene for the 1PP task showing five avatars located around a circle. One avatar was facing the participants $\left(180^{\circ}\right)$, two were on their right $\left(60^{\circ}\right.$ and $\left.120^{\circ}\right)$, and two on their left $\left(-60^{\circ}\right.$ and $120^{\circ}$ ). Participants were instructed to toss the ball to the avatar wearing a red T-shirt, which was located at $\pm 60^{\circ}$ or $\pm 120^{\circ}$ from their viewpoint (4 visual stimuli). Participants pressed a key with their right middle finger to toss the ball to the right, or their right index finger to toss the ball to the left. A fixation cross was presented during $1 \mathrm{~s}$ before each visual scene. The correct response here is to toss the ball to the right. (C) Example of a visual scene for the visual detection task. Visual stimuli were the same as for the 3PP taking task, except that all avatars wore a white T-shirt. Thirteen participants had to press a button with their right middle finger as soon as they detected a ball, or with their right index finger when they detected there was no ball, and vice versa for the other participants. A fixation cross was presented during $1 \mathrm{~s}$ before each visual scene. The correct response here is "yes", as there is a ball in the scene. (D) Example of a visual stimulus for the 3D objects mental rotation. Half of the participants pressed a button with their right middle finger if objects were identical, or with their right index finger if they were different, and vice versa for the others. The correct response here is "no", as the objects were different.

Figure 2. Influence of unilateral vestibular deafferentation on visuo-spatial perspective taking. (A) Mean response times for the 3PP taking task are plotted separately for the whole population of patients (left part), for the patients with left vestibular neurectomy (central part) and the patients with right vestibular neurectomy (right part) together with their age- and gender-matched control participants. Error bars represent the standard error of mean. * indicates statistical significance between the patients and their controls $(P<0.05)$. (B) Mean response times for the 3PP taking task are illustrated for the patients as a function of the vestibular pathology. (C) Box-and-whisker plots illustrate the percentage of 
456 errors in the whole sample of participants. The top and bottom ends of the whisker represent the $95^{\text {th }}$ and

${ }_{2}^{1} 4575^{\text {th }}$ percentiles of the distribution and the horizontal line inside the box represents the median. (D)

3458 Response times for the 1PP task and the visual detection task are plotted for the whole sample of patients.

4 Same conventions as for Parts C.

Figure 3. Influence of unilateral vestibular deafferentation on 3D objects mental rotation. (A) Mean response times for the mental rotation of $3 \mathrm{D}$ objects are plotted separately for the whole population of patients (left part), for the patients with left vestibular neurectomy (central part) and the patients with right vestibular neurectomy (right part) together with their age- and gender-matched control participants. Error bars represent the standard error of mean. (B) Box-and-whisker plots illustrate the percentage of errors in the whole sample of participants. The top and bottom ends of the whisker represent the $95^{\text {th }}$ and $5^{\text {th }}$ percentiles of the distribution and the horizontal line inside the box represents the median. 


\section{References}

1. Smith PF (2017) The vestibular system and cognition. Curr Opin Neurol 30:84-89. https://doi.org/10.1097/WCO.0000000000000403

2. Bigelow RT, Agrawal Y (2015) Vestibular involvement in cognition: Visuospatial ability, attention, executive function, and memory. J Vestib Res Equilib Orientat 25:73-89. https://doi.org/10.3233/VES-150544

3. Bigelow RT, Semenov YR, du Lac S, et al (2016) Vestibular vertigo and comorbid cognitive and psychiatric impairment: the 2008 National Health Interview Survey. J Neurol Neurosurg Psychiatry 87:367-372. https://doi.org/10.1136/jnnp-2015-310319

4. Popp P, Wulff M, Finke K, et al (2017) Cognitive deficits in patients with a chronic vestibular failure. J Neurol 264:554-563. https://doi.org/10.1007/s00415-016-8386-7

5. Péruch P, Borel L, Gaunet F, et al (1999) Spatial performance of unilateral vestibular defective patients in nonvisual versus visual navigation. J Vestib Res 9:37-47

6. Brandt T, Schautzer F, Hamilton DA, et al (2005) Vestibular loss causes hippocampal atrophy and impaired spatial memory in humans. Brain 128:2732-41

7. Allum JHJ, Langewitz W, Sleptsova M, et al (2017) Mental body transformation deficits in patients with chronic balance disorders. J Vestib Res Equilib Orientat 27:113-125. https://doi.org/10.3233/VES-170613

8. Grabherr L, Cuffel C, Guyot JP, Mast FW (2011) Mental transformation abilities in patients with unilateral and bilateral vestibular loss. Exp Brain Res 209:205-14. https://doi.org/10.1007/s00221-011-2535-0

9. Péruch P, Lopez C, Redon C, et al (2011) Vestibular information is necessary for maintaining metric properties of representational space: Evidence from mental imagery. Neuropsychologia 49:3136-3144

10. Candidi M, Micarelli A, Viziano A, et al (2013) Impaired mental rotation in benign paroxysmal positional vertigo and acute vestibular neuritis. Front Hum Neurosci 7:783. https://doi.org/10.3389/fnhum.2013.00783

11. Dieterich M, Bense S, Lutz S, et al (2003) Dominance for vestibular cortical function in the non-dominant hemisphere. Cereb Cortex 13:994-1007

12. Dieterich M, Kirsch V, Brandt T (2017) Right-sided dominance of the bilateral vestibular system in the upper brainstem and thalamus. $J$ Neurol 264:55-62. https://doi.org/10.1007/s00415-017-8453-8

13. Saj A, Honoré J, Bernard-Demanze L, et al (2013) Where is straight ahead to a patient with 
unilateral

vestibular

loss?

Cortex

49:1219-1228.

https://doi.org/10.1016/j.cortex.2012.05.019

14. Hüfner K, Hamilton DA, Kalla R, et al (2007) Spatial memory and hippocampal volume in humans with unilateral vestibular deafferentation. Hippocampus 17:471-85. https://doi.org/10.1002/hipo.20283

15. Kessler K, Thomson LA (2010) The embodied nature of spatial perspective taking: embodied transformation versus sensorimotor interference. Cognition 114:72-88. https://doi.org/10.1016/j.cognition.2009.08.015

16. Deroualle D, Borel L, Devèze A, Lopez C (2015) Changing perspective: The role of vestibular signals. Neuropsychologia 79, Part B:175-185. https://doi.org/10.1016/j.neuropsychologia.2015.08.022

17. David N, Bewernick BH, Cohen MX, et al (2006) Neural representations of self versus other: visual-spatial perspective taking and agency in a virtual ball-tossing game. J Cogn Neurosci 18:898-910

18. Becker-Bense S, Dieterich M, Buchholz H-G, et al (2014) The differential effects of acute right- vs. left-sided vestibular failure on brain metabolism. Brain Struct Funct 219:13551367. https://doi.org/10.1007/s00429-013-0573-z

19. Pavlidou A, Ferrè ER, Lopez C (2018) Vestibular stimulation makes people more egocentric. Cortex J Devoted Study Nerv Syst Behav 101:302-305. https://doi.org/10.1016/j.cortex.2017.12.005

20. Lopez C, Elzière M (2018) Out-of-body experience in vestibular disorders - A prospective study of 210 patients with dizziness. Cortex J Devoted Study Nerv Syst Behav 104:193206. https://doi.org/10.1016/j.cortex.2017.05.026

21. Macauda G, Moisa M, Mast FW, et al (2019) Shared neural mechanisms between imagined and perceived egocentric motion - A combined GVS and fMRI study. Cortex J Devoted Study Nerv Syst Behav 119:20-32. https://doi.org/10.1016/j.cortex.2019.04.004

22. Koos WT, Day JD, Matula C, Levy DI (1998) Neurotopographic considerations in the microsurgical treatment of small acoustic neurinomas. J Neurosurg 88:506-512. https://doi.org/10.3171/jns.1998.88.3.0506

23. Deroualle D, Lopez C (2014) Toward a vestibular contribution to social cognition. Front Integr Neurosci 8:16. https://doi.org/10.3389/fnint.2014.00016

24. Lenggenhager B, Lopez C, Blanke O (2008) Influence of galvanic vestibular stimulation on egocentric and object-based mental transformations. Exp Brain Res 184:211-21

25. Lopez C, Nakul E, Preuss N, et al (2018) Distorted own-body representations in patients 
with dizziness and during caloric vestibular stimulation. J Neurol 265:86-94. https://doi.org/10.1007/s00415-018-8906-8

26. Toupet M, Van Nechel C, Bozorg Grayeli A (2014) Influence of body laterality on recovery https://doi.org/10.1159/000360266

27. Bigelow RT, Semenov YR, Trevino C, et al (2015) Association between visuospatial ability and vestibular function in the Baltimore longitudinal study of aging. J Am Geriatr Soc 63:1837-1844. https://doi.org/10.1111/jgs.13609

28. Falconer CJ, Mast FW (2012) Balancing the mind: vestibular induced facilitation of egocentric mental transformations. Exp Psychol 59:332-339. https://doi.org/10.1027/1618-3169/a000161

29. Secora K, Emmorey K (2019) Social abilities and visual-spatial perspective-taking skill: deaf signers and hearing Nnonsigners. J Deaf Stud Deaf Educ. https://doi.org/10.1093/deafed/enz006

30. Stackman RW, Clark AS, Taube JS (2002) Hippocampal spatial representations require vestibular input. Hippocampus 12:291-303. https://doi.org/10.1002/hipo.1112 
A Virtual ball-tossing game: 3PP

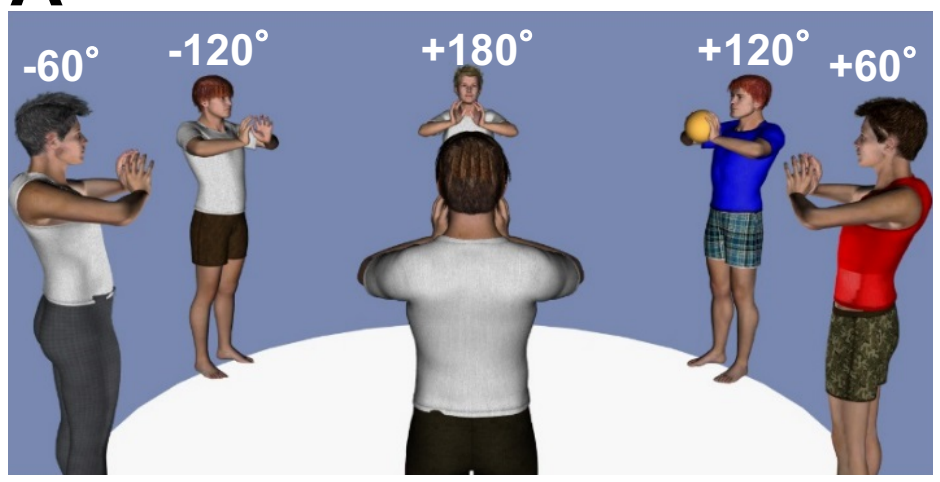

B Virtual ball-tossing game: 1PP

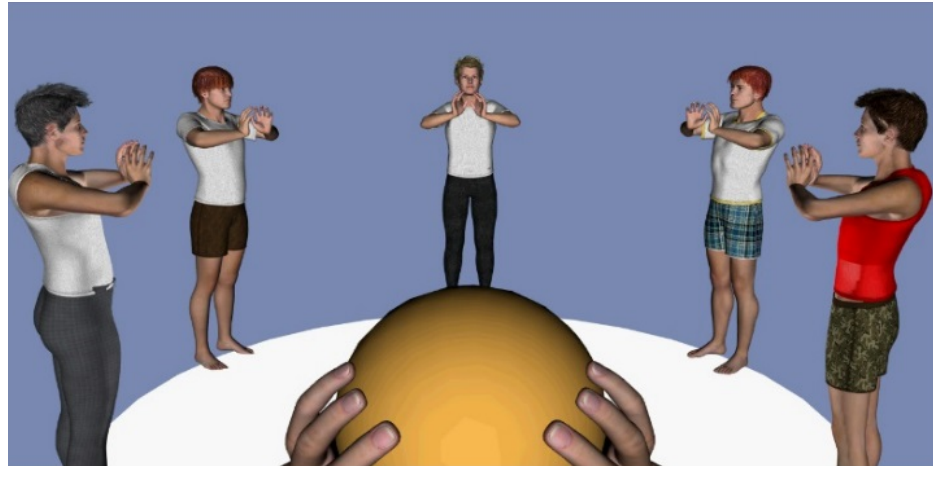

C Visual detection task: 1PP

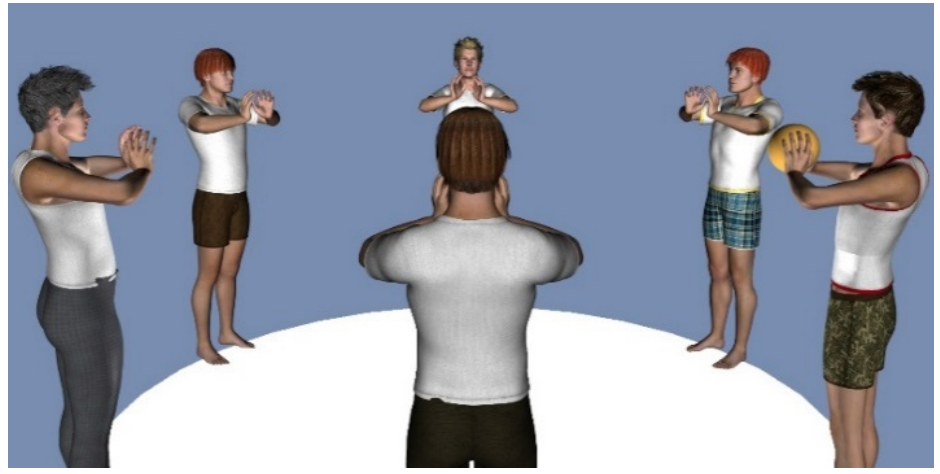

D 3D objects mental rotation task

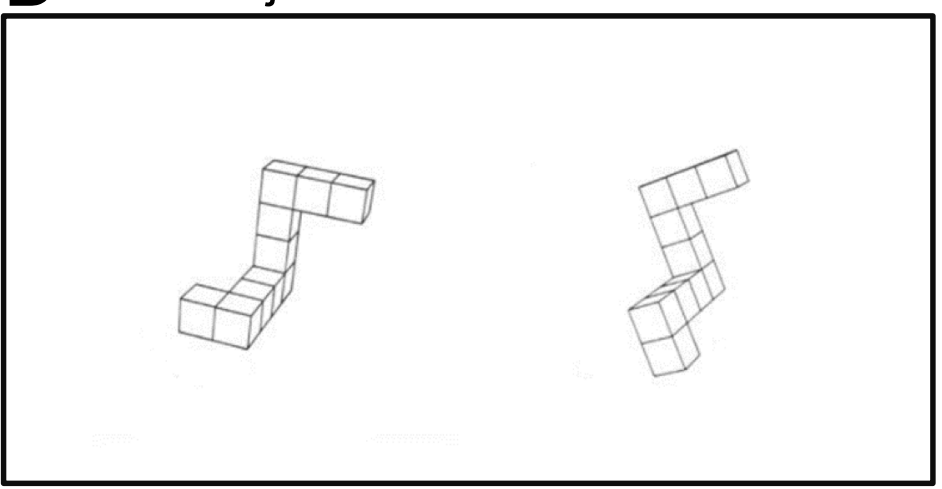



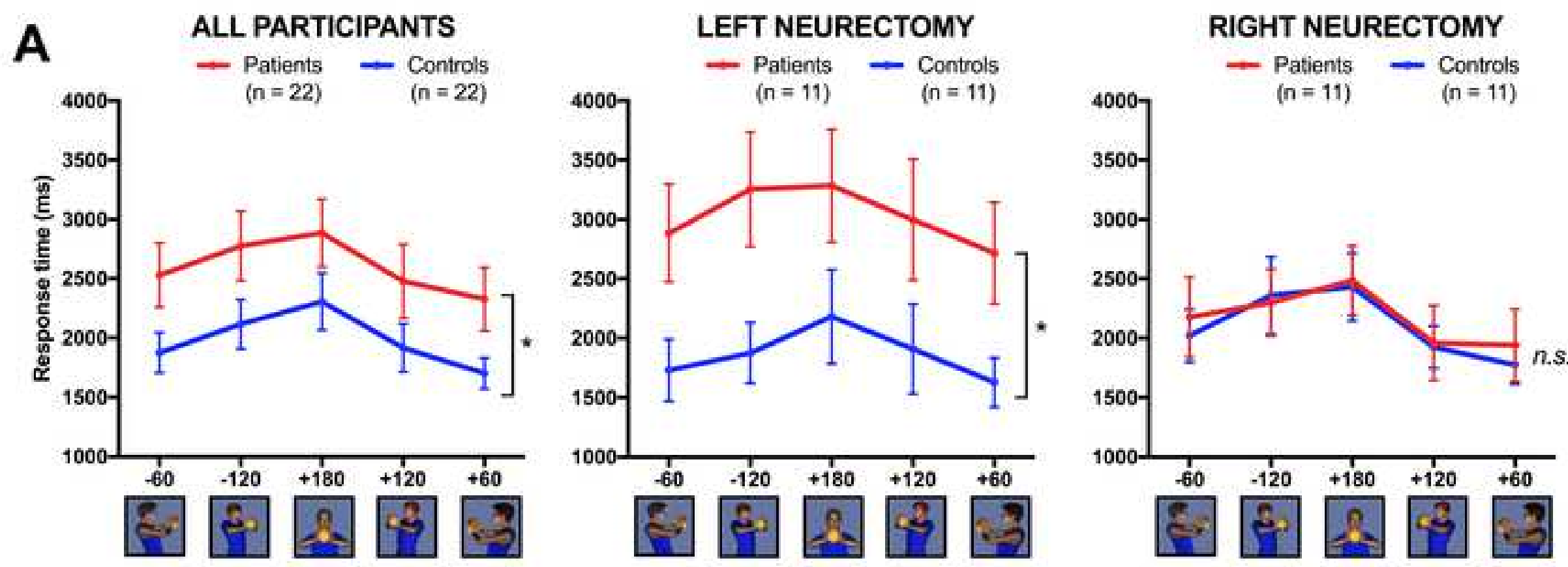

B

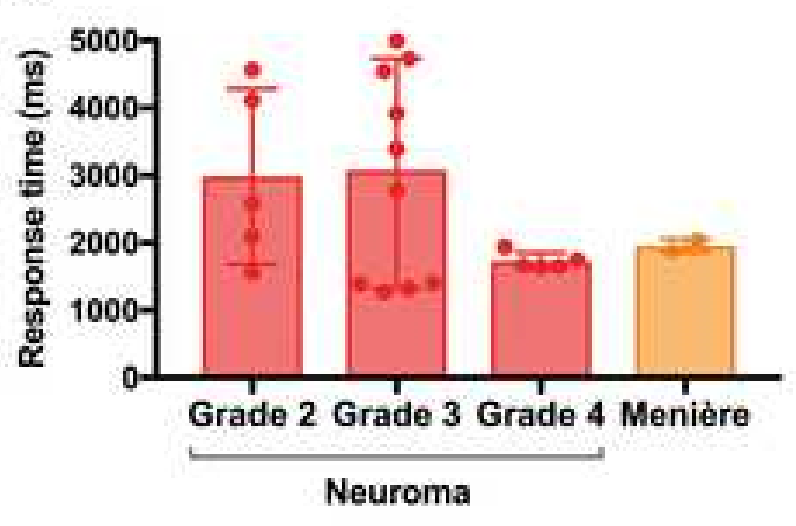

C

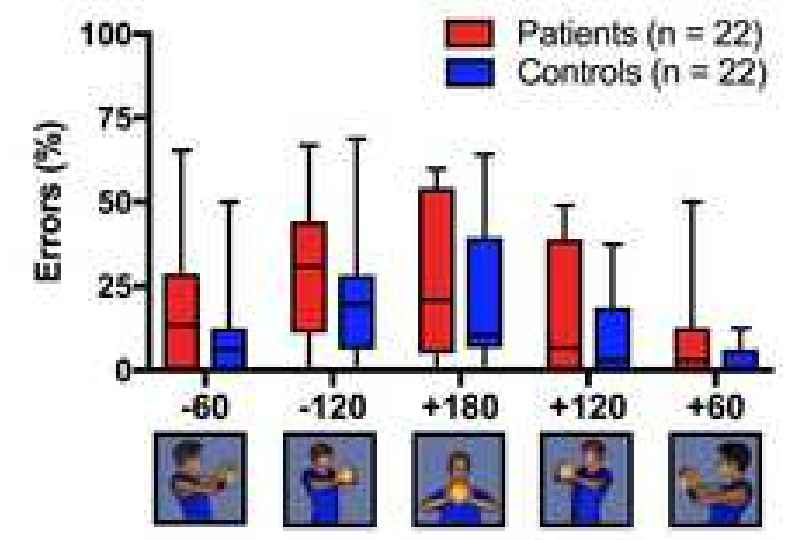

D

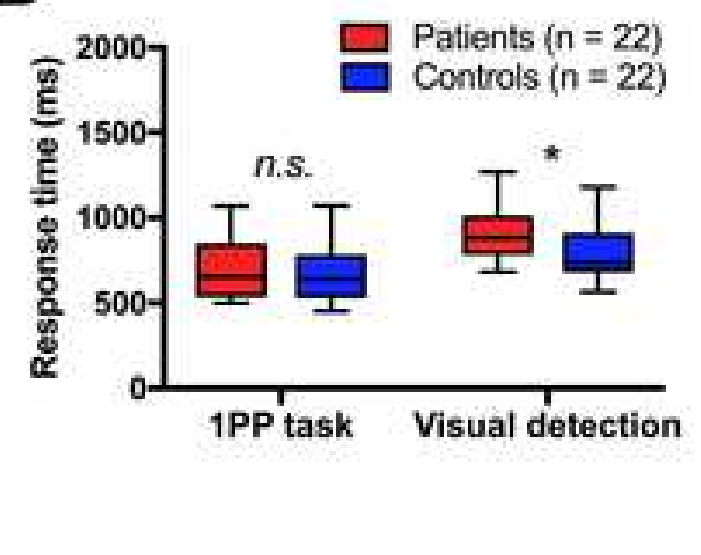



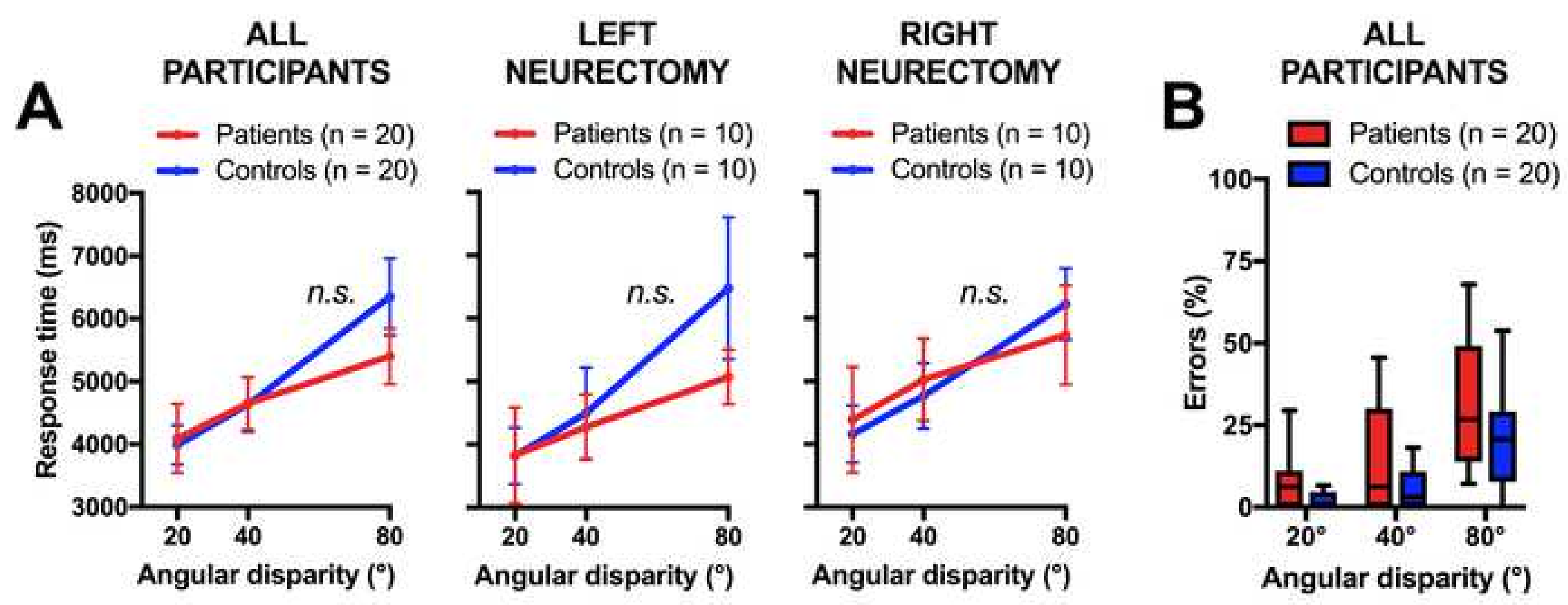


\begin{tabular}{|c|c|c|c|c|c|c|c|c|c|c|c|}
\hline & \multirow{2}{*}{$\begin{array}{c}\text { Age } \\
\text { (years) }\end{array}$} & \multirow[b]{2}{*}{ Gender } & \multirow[b]{2}{*}{ Diagnosis } & \multicolumn{4}{|c|}{ Examination at D-1 } & \multicolumn{4}{|c|}{ Examination at $\mathrm{D}+7$} \\
\hline & & & & $\begin{array}{c}\text { CVS } \\
\text { (\% deficit) }\end{array}$ & $\begin{array}{l}\text { VOR } \\
\text { (gain) }\end{array}$ & $\begin{array}{l}\text { VHIT } \\
\text { (gain) }^{\text {a }}\end{array}$ & $\begin{array}{l}\text { SVV } \\
\left({ }^{\circ}\right)^{b}\end{array}$ & $\begin{array}{c}\text { CVS } \\
\text { (\% deficit) }\end{array}$ & $\begin{array}{l}\text { VOR } \\
\text { (gain) }\end{array}$ & $\begin{array}{l}\text { VHIT } \\
\text { (gain) }^{\mathrm{a}}\end{array}$ & $\begin{array}{l}\text { SVV } \\
\left({ }^{\circ}\right)^{b}\end{array}$ \\
\hline $\begin{array}{c}\text { Left } \\
\text { neurectomy }\end{array}$ & $49.9 \pm 11.6$ & $\begin{array}{c}7 \text { women } \\
5 \text { men }\end{array}$ & $\begin{array}{l}4 \text { grade } 2 \\
6 \text { grade } 3 \\
2 \text { grade } 4\end{array}$ & $66.2 \pm 39.8$ & $0.36 \pm 0.14$ & $0.45 \pm 0.36$ & $-2.3 \pm 2.4$ & $95.6 \pm 11.7$ & $0.43 \pm 0.21$ & $0.38 \pm 0.41$ & $-7.1 \pm 4.0$ \\
\hline $\begin{array}{c}\text { Right } \\
\text { neurectomy }\end{array}$ & $53.0 \pm 14.0$ & $\begin{array}{c}6 \text { women } \\
5 \text { men }\end{array}$ & $\begin{array}{c}3 \text { grade } 2 \\
3 \text { grade } 3 \\
3 \text { grade } 4 \\
2 \mathrm{MD}\end{array}$ & $41.9 \pm 34.3$ & $0.44 \pm 0.22$ & $0.69 \pm 0.36$ & $0.1 \pm 1.0$ & $80.0 \pm 25.2$ & $0.41 \pm 0.22$ & $0.25 \pm 0.28$ & $-6.2 \pm 4.2$ \\
\hline & $P=0.57^{\mathrm{c}}$ & $P=0.86^{\mathrm{d}}$ & $P=0.63^{\mathrm{e}}$ & $P=0.19^{\mathrm{c}}$ & $P=0.35^{\mathrm{c}}$ & $P=0.18^{\mathrm{c}}$ & $P=0.01^{\mathrm{c}}$ & $P=0.16^{\mathrm{c}}$ & $P=0.90^{\mathrm{c}}$ & $P=0.56^{\mathrm{c}}$ & $P=0.63^{\mathrm{c}}$ \\
\hline
\end{tabular}

Table 1. Comparison of clinical data in patients with left and right vestibular neurectomy. Means \pm standard deviations are reported before (D-1) and after (D+7) surgery for each group of patients. MD: Menière's disease, CVS: caloric vestibular stimulation, VOR: vestibulo-ocular reflex, VHIT: video head impulse test, SVV: subjective visual vertical.

\footnotetext{
${ }^{\text {a }}$ Gain for the lateral semicircular canal on the side of the disease

${ }^{\mathrm{b}}$ Negatives signs indicate that the subjective visual vertical was tilted to the side of the disease (ipsilesional tilt)

${ }^{\mathrm{c}}$ Independent-samples t-tests

${ }^{\mathrm{d}}$ Chi-square

${ }^{\text {e }}$ Chi-square for the grade of neuroma
} 


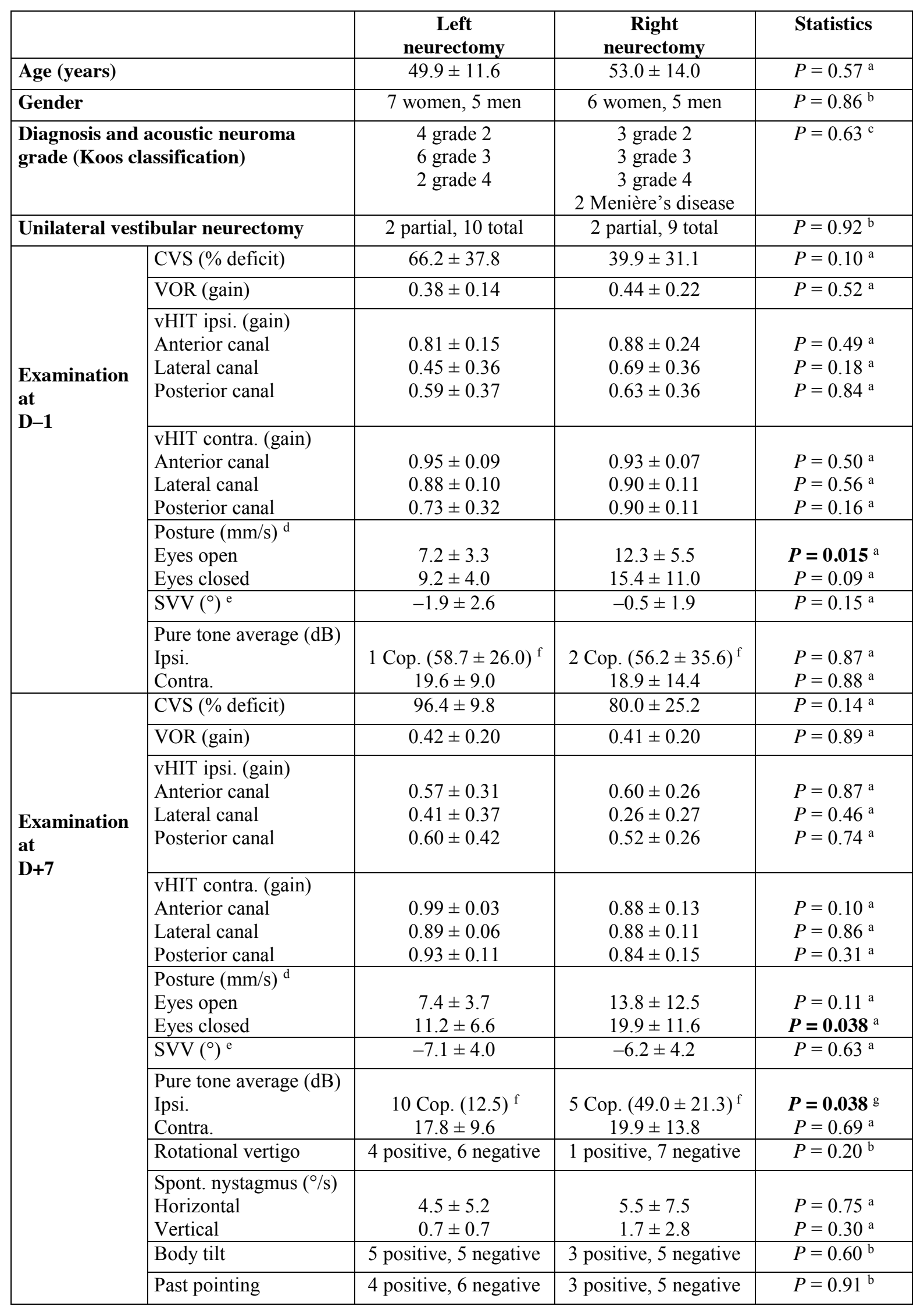

Table 1. Comparison of clinical data in patients with left and right vestibular neurectomy. Means \pm standard deviations are reported before (D-1) and after (D+7) surgery for each group of patients. CVS: caloric vestibular stimulation, VOR:

\footnotetext{
${ }^{a}$ Independent-samples t-tests

${ }^{\mathrm{b}}$ Chi-square

${ }^{\mathrm{c}}$ Chi-square for the grade of neuroma

${ }^{d}$ Average velocity of the center of foot pressure measured on a posturography platform with a stable support surface

${ }^{\mathrm{e}}$ Negatives signs indicate an ipsilesional tilt of the subjective visual vertical

${ }^{\mathrm{f}}$ The number of patients with cophosis (Cop.) is indicated; the mean hearing loss in the other patients without cophosis is indicated in brackets

${ }^{\mathrm{g}}$ Chi-square for the number of patients with cophosis
} 
vestibulo-ocular reflex, VHIT: video head impulse test, SVV: subjective visual vertical, ipsi.: ipsilesional ear, contra.: contralesional ear, Cop.: cophosis. 